\title{
Polysaccharides from the South African medicinal plant Artemisia afra: Structure and activity studies
}

Paula Marie Braünlich, Kari Tvete Inngjerdingen, Marit Inngjerdingen, Quinton Johnson, Berit Smestad Paulsen and Wilfred Mabusela

\begin{abstract}
Artemisia afra (Jacq. Ex. Willd), is an indigenous plant in South Africa and other parts of the African continent, where it is used as traditional medicine mostly for respiratory conditions. The objective of this study was to investigate the structural features of the polysaccharides from the leaves of this plant, as well as the biological activities of the polysaccharide fractions against the complement assay. Leaves of Artemisia afra were extracted sequentially with organic solvents (dichloromethane and methanol), 50\% aqueous ethanol, and water at 50 and $100{ }^{\circ} \mathrm{C}$ respectively. The polysaccharide extracts were fractionated by ion exchange chromatography and the resulting fractions were tested for biological activity against the complement fixation assay. Active fractions were further fractionated using gel filtration. Monosaccharide compositions and linkage analyses were determined for the relevant fractions. Polysaccharides were shown to be of the pectin type, and largely contain arabinogalactan, rhamnogalacturonan and homogalacturonan structural features. The presence of arabinogalactan type II features as suggested by methylation analysis was further confirmed by the ready precipitation of the relevant polysaccharides with the Yariv reagent. An unusual feature of some of these polysaccharides was the presence of relatively high levels of xylose as one of its monosaccharide constituents. Purified polysaccharide fractions were shown to possess higher biological activity than the selected standard in the complement assay. Digestion of these polysaccharides with an endo-polygalacturonase enzyme resulted in polymers with lower molecular weights as expected, but still with biological activity which exceeded that of the standard. Thus on the basis of these studies it may be suggested that immunomodulating properties probably contribute significantly to the health-promoting effects of this medicinal plant.
\end{abstract}

\section{Introduction}

Artemisia afra is a plant which is used traditionally in South Africa against a variety of indications. Some of these include internal worm infestation, cough, cold, fever, colic, headache, loss of appetite, pain in the ear and malaria [15]. Locally it is commonly known as African wormwood, while it also has such vernacular names as "umhlonyane" (Xhosa, Zulu), "lengana" (Sotho, Tswana) and "wildeals" (Afrikaans) The most common way to use 
the plant is to inhale the steam after boiling the leaves in water, although it may also be taken as a tea infusion [31]. Other uses include putting the leaves in the socks to prevent sweating; they are also used against hemorrhoids, diabetes, digestive problems, on wounds and as insecticide [29]. Artemisia afra is one of the most used plants in South Africa, and other African countries as well; the plant has been the object of various studies over the recent years, although focus has mainly been on essential oils and low molecular weight compounds, mostly for their association with antimicrobial activity. According to the SciFinder Scholar Chemical Abstracts database, the first scientific report on essential oils of A. afra was published in 1930 [25] and since then several articles have emerged. A significant number of studies have focused on the variation of phyto-chemical composition as influenced by geographical location, method of isolation as well as plant material drying methods. Geographical location appears to be characterized by which of the constituents occur as the major components in the oil. For example, in a study conducted by Oyedeji et al. [18], of plant materials collected from three different provinces of South Africa, it was shown that each of the populations was either dominated by 1,8-cineole, $\alpha$ - or $\beta$-thujone, or camphor. In a similar study by Viljoen et al. [28], variations had also been observed, although the spread of major constituents was of a different profile. Nevertheless there is agreement that the $A$ afra species may be subdivided into more than one chemotype. In terms of isolation methods, it has been shown that low temperature methods often reveal a broader diversity of constituents than the traditional hydrodistillation method [2]. An investigation into the influence of drying methods revealed that there was no significant loss of volatile constituents during air-drying, oven-drying or sun-drying except for the marginal loss of sesquiterpenoids during sun-drying [1]. An interesting observation was made when microwave drying was included as an additional option [3], whereby it was found that the latter method resulted in complete removal of 1,8 -cineole and $\beta$-thujone, as well as the poorest yield of the four drying methods. On the other hand, qualitatively it displayed the widest diversity of essential constituents with sesquiterpenoids being the predominant class among the terpenes.

Non-volatile constituents, which are largely extracted with organic solvents, have been isolated over the years and shown to be mostly terpenoids and flavonoids [15]. As one of those multi-purpose medicinal plants, A. afra has also been investigated for potential cardioprotective and antidiabetic cytotoxic properties, for which results were shown to be positive in animal experiments [11,12,22]. Cytotoxicity studies of an ethanol extract against HeLa cancer cells followed by a bio-assay guided fractionation led to the isolation of a sesquiterpene lactone, known as isoalantolactone, as the main bioactive compound $[21,27]$. The plant has also been shown to contain products that indicate it to have efficacy against experimental tuberculosis [17]. Since water extraction is one of the most common ways of preparation of products used from the plant, it is interesting that little has been done on the compounds soluble only in water, among these, the polysaccharides. Recent studies by various groups have shown that polysaccharides may play an important role in illnesses related to the immune system [19,30,32]. We have recently described the immunomodulating activity of pectic polysaccharides from the South African medicinal plant Lessertia frutescens, (syn. Sutherlandia frutescens), [36]. Hence it was of interest to 
us to study the polysaccharides present in the above-ground parts of Artemisia afra and also investigate possible effects in immunomodulating assays.

\section{Experimental}

\subsection{Plant material}

The plant material, Artemisia afra Jacq. Ex Willd., Compositae, was collected from the botanical gardens of the Montague Museum (Western Cape, South Africa) and consists of the stalks and leaves of the plant; its identity was authenticated by Frans Weitz, a plant systematist from the Department of Biodiversity and Conservation Biology, University of the Western Cape, South Africa. (Voucher specimen number 6639 is deposited in the herbarium at the University of the Western Cape, Cape Town, South Africa.)

\subsection{Preparation of the polysaccharide fractions}

Prior to the extraction of the polysaccharides, the plant material was extracted with organic solvents to remove lipophilic and low molecular weight substances. This was performed in a Soxhlet apparatus, successively with dichloromethane and methanol. The residue was then subjected to extraction with $50 \%$ ethanol in water, followed by extraction with water at $50^{\circ} \mathrm{C}$ and finally water of $100^{\circ} \mathrm{C}$. The $50 \%$ ethanol extract was first evaporated to remove all ethanol present, and then filtered to remove material not soluble in water. This extract was then treated in the same way as the two water extracts described below. All fractions were subjected to ultrafiltration using a PALL suspended screen channel Ultrasett with cut off $5 \mathrm{kD}$. The high molecular weight fractions were then applied onto an anion exchange column, ANX Sepharose 4 Fast Flow, 5x20cm, in chloride form. The neutral polysaccharides were eluted with distilled water, and the acidic ones with a NaCl-gradient, o-1.5 M NaCl. The eluent flow rate was $2 \mathrm{~mL} / \mathrm{min}$. Fractions of $10 \mathrm{~mL}$ were collected in a Pharmacia LKB SuperFrac fraction collector, and they were monitored for the content of carbohydrate by the phenol-sulfuric acid method [8]. The fractions which were rich in carbohydrates and with similar migratory behavior were pooled, dialyzed using a membrane of cut off $3500 \mathrm{D}$, against distilled water in order to remove $\mathrm{NaCl}$, and then freeze-dried. All fractions were tested for bioactivity using the complement assay as described below. The fractions that proved to be bioactive after anion exchange chromatography (one from the $50 \%$ ethanol in water extract, one from the $50^{\circ} \mathrm{C}$ water extract and two from the $100^{\circ} \mathrm{C}$ water, designated as 50\% $\mathrm{EtOH}, 50 \mathrm{~W}, 100 \mathrm{~W} 1$ and $100 \mathrm{~W} 2$ respectively), were all further fractionated by gel filtration chromatography using a Sephacryl S-200 matrix (MW range 5$250 \mathrm{kDa}$ ), Pharmacia Fine Chemicals, 3x70cm. The eluent was $0.3 \mathrm{M}$ sodium acetate; flow rate $1 \mathrm{~mL} / \mathrm{min}$ and fractions of $5 \mathrm{~mL}$ were collected and tested with the phenol-sulfuric acid method as described above. The fractions that were rich in carbohydrates and with similar migratory behavior were pooled, dialyzed against a cut off of $3500 \mathrm{D}$, freeze-dried and analyzed for bioactivity using the complement assay described below. All fractions with bioactivity, as obtained after gel filtration chromatography, were objects for more detailed structural and bioactivity studies. 


\subsection{Determination of the monosaccharide composition}

The monosaccharide compositions of the extracts and purified fractions were determined by gas chromatography of the trimethylsilylated (TMS) derivatives of the methyl glycosides obtained after methanolysis of the polymers with $3 \mathrm{M}$ hydrochloric acid in anhydrous methanol for $24 \mathrm{~h}$ at $80^{\circ} \mathrm{C}$ [4-6]. Mannitol was used as an internal standard. The TMS derivatives were analyzed by capillary gas chromatography on a Focus GC (Thermo Scientific, Milan, Italy) and the amount of each monosaccharide present was determined based on standard curves of each monosaccharide.

\subsection{Molecular weight determination}

The homogeneity and molecular weights of the purified polysaccharide fractions were determined by size exclusion chromatography on a Superose 6 prepacked column (Amersham Biosciences) combined with the Äkta system (FPLC, Pharmacia Äkta, Amersham Pharmacia Biotech). Dextran polymers (Pharmacia) B512 (5.6 kDa), T8360 (19 $\mathrm{kDa})$, T250 (233 kDa) and T500 (475 kDa) were used as calibration standards. Approximately $5 \mathrm{mg}$ of the samples were dissolved in $2 \mathrm{~mL}$ of $10 \mathrm{mM} \mathrm{NaCl}$ buffer, the resulting solution was filtered through a Millipore filter $(0.45 \mu \mathrm{m})$ and then applied to the column. The samples were eluted with $0.3 \mathrm{M}$ sodium acetate at $\mathrm{pH} 5.2$, with a speed of 0.5 $\mathrm{mL} / \mathrm{min}$, collecting $2 \mathrm{~mL}$ fractions. The eluent was monitored with a Shimadzu RI detector. The retention volume of the main peak of each sample was converted to molecular weight based on a standard curve prepared on the basis of the standards given above.

\subsection{Precipitation with the Yariv $\beta$-glucosyl reagent}

Precipitation with the Yariv $\beta$-glucosyl reagent was performed on the samples as described by van Holst and Clarke [26]. The Yariv $\beta$-glucosyl reagent forms a colored precipitate with compounds containing certain types of AG-II structures. A solution of Arabic gum in water $(1 \mathrm{mg} / \mathrm{mL})$ was used as a positive control.

\subsection{Determination of glycosidic linkages}

Glycosidic linkage determination was performed through methylation studies. The fractions most active in the complement assay from each extract were selected for linkage determination. Prior to methylation, the uronic acids were reduced with $\mathrm{NaBD}_{4}$ to their corresponding neutral sugars, dideuterated in position 6. After reduction of the polymers, methylation, hydrolysis, reduction using sodium borodeuteride and acetylation were carried out [13]. The derivatives were analyzed by GC-MS using a GCMS-QP2010 (Shimadzu) in which was attached a Restek Rxi-5MS capillary column (30 m; $0.25 \mathrm{~mm}$ i.d.; $0.25 \mu \mathrm{m}$ film). The injector temperature was $280^{\circ} \mathrm{C}$, the ion source temperature 200 ${ }^{\circ} \mathrm{C}$ and the interface temperature $300^{\circ} \mathrm{C}$. The column temperature was $80^{\circ} \mathrm{C}$ when injected, then increased at $10{ }^{\circ} \mathrm{C} / \mathrm{min}$ to $140{ }^{\circ} \mathrm{C}$, followed by $4{ }^{\circ} \mathrm{C} / \mathrm{min}$ to $210^{\circ} \mathrm{C}$, and then $20{ }^{\circ} \mathrm{C} / \mathrm{min}$ to $300{ }^{\circ} \mathrm{C}$. Helium was the carrier gas (pressure control: $80 \mathrm{kPa}$.) The compounds represented by each peak were characterized by their retention times and interpretation of their characteristic mass spectra. The estimation of the relative amounts of each linkage type was related to the total amount of each monosaccharide type as determined by methanolysis. Effective carbon-response factors were applied for quantification [23]. 


\subsection{Complement fixation assay}

The complement system is an important part of the innate immune system which also cooperates with the adaptive immune system in many ways [9]. The complement system does, among other things, play a direct role in defense, such as primary defense against bacterial invasions and viral infections. Complement fixating activity of polysaccharides from plants has previously been used as an indicator for the effect on the immune system [16]. The complement fixation test is based on inhibition of haemolysis of antibody sensitized sheep red blood cells (SRBC) by human sera as described by Michaelsen et al. (Method A) [16,24], using PMII, a polysaccharide from Plantago major, as the positive control. The indicator system in the assay is inhibition of haemolysis induced by human complement. Samples showing inhibition in the assay is thus having a direct effect on the human immune system. Inhibition of lysis induced by the test samples was calculated by the formula [(Acontrol - Atest)/Acontrol] $\times 100 \%$, A is absorbance at $415 \mathrm{~nm}$. From these data, a dose-response curve was created to calculate the concentration of test sample giving $50 \%$ inhibition of lysis $\left(\mathrm{ICH}_{50}\right)$. A low $\mathrm{ICH}_{50}$ value means a high complement fixation activity. All samples were analyzed in triplicate and the data shown is a typical example of results obtained.

\subsection{Degradation of the samples with endo-polygalacturonase}

Polysaccharide material ( $5 \mathrm{mg}$ ) was dissolved in $3 \mathrm{~mL} \mathrm{o.1} \mathrm{M} \mathrm{NaOH}$ for de-esterification. The solution was left for $2 \mathrm{~h}$ and neutralized with acetic acid. Sodium acetate buffer (10 mL), at $\mathrm{pH} 4.2$ and 1 drop toluene were added followed by $60 \mu \mathrm{L}$ of endo-polygalacturonase M2 (Megazyme). The mixture was then kept at $37^{\circ} \mathrm{C}$ for $72 \mathrm{~h}$. After boiling to denature the enzyme, the mixture was filtered through a Millipore filter $0.45 \mu \mathrm{m}$ and the filtrate was concentrated to $1 \mathrm{~mL}$ prior to application on a PD-10 column. The PD-10 column was then eluted as described by the manufacturer (Pharmacia), the recovered high molecular weight material was freeze dried and subjected to monosaccharide determination and the complement assay.

\section{Results and Discussion}

The dried plant material (406 g) was extracted as described. The fractionation scheme is given in Fig. 1 below:

The yield obtained, of the fractions showing high activity in the complement assay after anion exchange chromatography and gel filtration is given in Table 1 . The 50\% EtOH and $50 \mathrm{~W}$ extracts gave only one bioactive fraction each after purification by anion exchange chromatography, while the $100 \mathrm{~W}$ fraction gave 2 bioactive fractions. The bioactive, purified fractions were denominated as $50 \% \mathrm{EtOH}, 50 \mathrm{~W}, 100 \mathrm{~W} 1$ and $100 \mathrm{~W} 2$ and were used for further studies. 


\subsection{Structural analyses of the polysaccharides from A. afra}

Monosaccharide analyses of the four fractions gave results typical for pectic polysaccharides (Table 2).

All fractions, apart from 100W2 have high contents of arabinose and galactose, indicating the presence of arabinogalactans, polymers which are commonly present in pectins as side chains to the main core. Galacturonic acid is present in fairly high amounts, and given also the content of rhamnose observed, the polymers may contain a main core consisting of a rhamnogalacturonan backbone linked with longer chains of homogalaturonan. The other monosaccharides detected are also usually present in pectins [32]. The most striking feature among these, is the relatively high amount of xylose present, which may have an impact on the bioactivity, and this will be discussed further below. The molecular weights of these four fractions were $69,88,43$ and $10 \mathrm{kD}$ respectively as related to dextrans. This may not be the real MWs as the folding, and thus the hydrodynamic volumes of the polysaccharide fractions in our studies may be different from those of dextrans. After degradation of the polymers with the enzyme endopolygalacturonanase, the most bioactive fractions thus obtained did also reveal the presence of small amounts of 4-O-methylglucuronic acid, also frequently found in pectins as terminal units [32].

The types of linkages present in the four bioactive native fractions obtained were determined on the basis of methylation analysis (Table 3). 


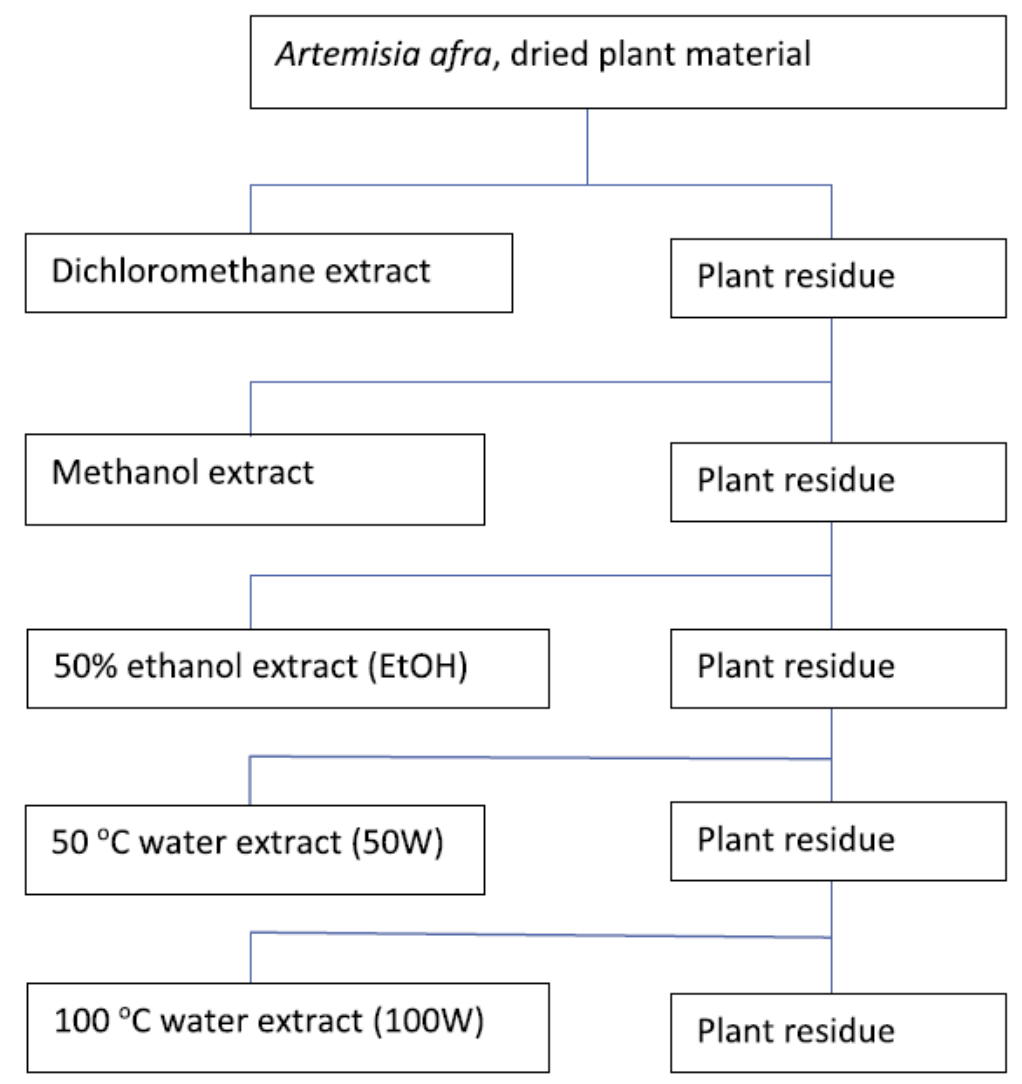

Fig. 1. Fractionation scheme of Artemisia afra.

Table 1

Yield (based on dry plant material) of the purified bioactive fractions obtained after separation by anion exchange and gel chromatography.

\begin{tabular}{llll}
\hline $\begin{array}{l}\text { Original plant } \\
\text { material, 406 g }\end{array}$ & Chromatography methods (yield in mg) & \\
\hline Fractions & $\begin{array}{l}\text { Anion } \\
\text { exchange }\end{array}$ & $\begin{array}{l}\text { Gel filtration } \\
\text { chromatography }\end{array}$ & $\begin{array}{l}\% \text { of start } \\
\text { material }\end{array}$ \\
$50 \% \mathrm{EtOH}$ & $807 \mathrm{mg}$ & $207 \mathrm{mg}$ & $0,05 \%$ \\
$50 \mathrm{~W}$ & $243 \mathrm{mg}$ & $60 \mathrm{mg}$ & $0,014 \%$ \\
$100 \mathrm{~W} 1$ & $1615 \mathrm{mg}$ & $355 \mathrm{mg}$ & $0,09 \%$ \\
$100 \mathrm{~W} 2$ & $1080 \mathrm{mg}$ & $712 \mathrm{mg}$ & $0,18 \%$ \\
\hline
\end{tabular}

Table 2

Monosaccharide composition of the most bioactive fractions, after separation by anion-exchange and gel filtration chromatography, and the high molecular weight fraction obtained after treatment with endo polygalacturonase (E) followed by purification on PD10.

\begin{tabular}{|c|c|c|c|c|c|c|c|c|}
\hline Fraction & $50 \% \mathrm{EtOH}$ & $50 \%$ EtOHE & $50 \mathrm{~W}$ & 50WE & 100W1 & 100W1E & 100W2 & $100 \mathrm{~W} 2 \mathrm{E}$ \\
\hline \multicolumn{9}{|l|}{ Monosaccharide } \\
\hline Arabinose & 19,0 & 15,9 & 12,6 & 12,2 & 10,6 & 9,5 & 3,5 & 4,7 \\
\hline Rhamnose & 7,9 & 8,7 & 5,6 & 6,2 & 11,9 & 14,2 & 5,7 & 9,1 \\
\hline Fucose & 0,4 & 0,2 & 0,4 & 0,4 & 0,6 & 0,6 & 0,6 & 1,2 \\
\hline Xylose & 7,8 & 4,5 & 15,1 & 10,5 & 4,4 & 4,5 & 1,3 & 2,1 \\
\hline Mannose & 3,2 & 3,1 & 3,1 & 2,2 & 4,7 & 1,3 & 7,5 & 2,8 \\
\hline Glucose & 1,8 & 2,8 & 3,2 & 2,8 & 3,8 & 3,6 & 9,5 & 6,6 \\
\hline Glucuronic acid & 4,4 & 4,3 & 8 & 5,7 & 5,7 & 4,2 & 4,5 & 2,2 \\
\hline Galactose & 33,4 & 34,7 & 26,3 & 27,3 & 17,3 & 16,6 & 5,8 & 7,3 \\
\hline Galacturonic acid & 22,1 & 22,9 & 25,7 & 30,4 & 41,0 & 44,8 & 61,6 & 63,2 \\
\hline 4-O-Me-GlcA & tr. & 2,9 & tr. & 2,3 & tr. & 0,7 & tr. & 0,8 \\
\hline
\end{tabular}


Table 3

Linkage analyses of the bioactive polysaccharides. The linkage types for the monosaccharides not mentioned were not detected. The linkages for fucose and mannose are not included.

\begin{tabular}{|c|c|c|c|c|c|}
\hline & Fraction & $50 \% \mathrm{EtOH}$ & $50 \mathrm{~W}$ & 100W1 & 100W2 \\
\hline Monosaccharide & Linkage type & \multicolumn{4}{|c|}{ Ratio of the different linkage types } \\
\hline \multirow[t]{5}{*}{ Arabinose } & $\mathrm{T} f$ & 10,1 & 4,6 & 5 & 1,4 \\
\hline & $1,3 f$ & 1,5 & 0,6 & 0,6 & tr. \\
\hline & $1,5 f$ & 5,9 & 4,3 & 2,8 & 1,3 \\
\hline & $1,3,5 f$ & 0,5 & 0,4 & 2,2 & 0,8 \\
\hline & $1,2,5 f$ & 1 & 2,7 & n.d. & n.d. \\
\hline \multirow[t]{3}{*}{ Rhamnose } & $\mathrm{T} p$ & 2,3 & 3,4 & 1,9 & 3,5 \\
\hline & $1,2 p$ & tr. & n.d. & 5,4 & 1,2 \\
\hline & $1,2,4 p$ & 5,6 & 2,2 & 4,6 & 1 \\
\hline \multirow[t]{2}{*}{ Xylose } & $\mathrm{T} p$ & 1,2 & 3,1 & 4,4 & 1,3 \\
\hline & $1,4 p$ & 6,6 & 12 & n.d & n.d. \\
\hline \multirow[t]{3}{*}{ Glucose } & $\mathrm{T} p$ & tr. & tr. & 1,1 & 1,9 \\
\hline & $1,4 p$ & 1,4 & 3,1 & 2,5 & 7,6 \\
\hline & $1,4,6 p$ & 0,4 & 0,1 & 0,2 & n.d. \\
\hline Glucuronic acid & $\mathrm{T} p$ & 4,4 & 8 & 5,7 & n.d. \\
\hline \multirow[t]{5}{*}{ Galactose } & $\mathrm{T} p$ & 7,1 & 7,7 & 6,4 & tr. \\
\hline & $1,3 p$ & 3 & 2,3 & 1,9 & 1,9 \\
\hline & $1,6 p$ & 10,4 & 8,4 & 5 & n.d. \\
\hline & $1,3,6 p$ & 11,7 & 7,1 & 3,5 & 2,5 \\
\hline & $1,3,4,6 p$ & 1,2 & 0,8 & 0,5 & 1,4 \\
\hline \multirow[t]{4}{*}{ Galacturonic acid } & $\mathrm{T} p$ & n.d. & tr. & n.d. & 10,1 \\
\hline & $1,4 p$ & 20,2 & 24,5 & 37,3 & 51,5 \\
\hline & $1,3,4 p$ & 1,4 & 1,1 & 2,5 & n.d. \\
\hline & $1,2,4 p$ & 0,5 & 0,1 & 1,2 & n.d. \\
\hline
\end{tabular}

$\mathrm{T}=$ non-reducing terminal unit; $f=$ furanose; $p=$ pyranose; $\operatorname{tr}=$ trace; n.d. $=$ not determined.

The fractions were all rich in 1,4-linked galacturonic acid residues, and all except Fr. 100W2 contained significant amounts of galacturonic acid residues that additionally were branched in either position 2 or 3 . These are features which have recently been shown to be present in polysaccharides from other medicinal plants, e.g. Combretum glutinosum, where it is believed that xylose units are appended to such branch positions [10]. It is thus interesting to note that terminal and 1,4-linked xylose units are also found in the polysaccharides from $A$. afra. Rhamnose, often part of the main RG I core as 1,2 linked units, is mainly found in Fr. 100W1 and 100W2; in all fractions rhamnose units linked both in positions 2 and 4 are present, indicating that the expected 1,2 linkages present in the RGI region are concomitantly carrying branches at position 4 . These features are also common in RGI units from other plant polysaccharides [32].

Galactose normally constitutes the main core in arabinogalactans I and II. With the exception of FR. 100W2, the galactose units were mostly 1,6-linked with significant branching at position 3, thus providing the important side-chains often observed in AGII structures [26]. The 
arabinose units, in their furanose form, are largely present as non-reducing terminal and 1,5linked units in the peripheral parts of these side-chains.

The Yariv reagent has the ability to precipitate polysaccharides containing arabinogalactan type II. Depending on the length of the galactose chain, a precipitate will form if galactose units contain branching at positions 3 and $6[26,14,20]$.

Since linkage analyses revealed the presence of galactose units which are linked at positions 3 and 6, the four polysaccharide fractions were tested against the Yariv reagent. Fractions 50\% EtOH, 50W and 100W1 precipitated with Yariv reagent, while fraction 100W2 did not, although 1,3,6-linkages were present. The specification of the Yariv reagent for binding the AGPII containing the 1,3,6-linked galactoses, remained unknown for a long time. However recently, following a systematic investigation by Kitazawa et al. [14], it was concluded that the $\beta$-1,3-galactan chain should be at least 5 units long in order to bind with the Yariv reagent, and the presence of 7 units was needed to form cross linkages and thus produce a precipitate. They also concluded that 1,6-linked chains did not bind the Yariv reagent. The results of our studies related to the Yariv test are displayed in Fig. 2.

Based on the above theory, it was concluded that samples 50\% EtOH, 50W and 100W1 all contain sequences consisting of at least 7 units of $\beta-1,3$-linked galactose units in their arabinogalactan chain, while fraction 100W2, although containing 1,3-linked and 1,3,6-linked galactose units, probably had shorter chains of the $\beta$-1,3-linked units, and hence was unable to form a precipitate.

Polysaccharides from other Artemisia species have also been studied. Yamada et al. [33-35] showed that the polysaccharides from the leaves of the Japanese medicinal plant, Artemisia princeps, contain immunomodulatory polysaccharides of the pectic type. The polysaccharides were shown to have a typical rhamnogalacturonan type I backbone, in which position 4 of the 1,2-linked rhamnose units were substituted with arabinogalactan type II sidechains, i.e. have galactose being $1,3^{-} ; 1,6-$ and 1,3,6-linked, with arabinofuranose units attached at the peripheral parts of these side chains. 


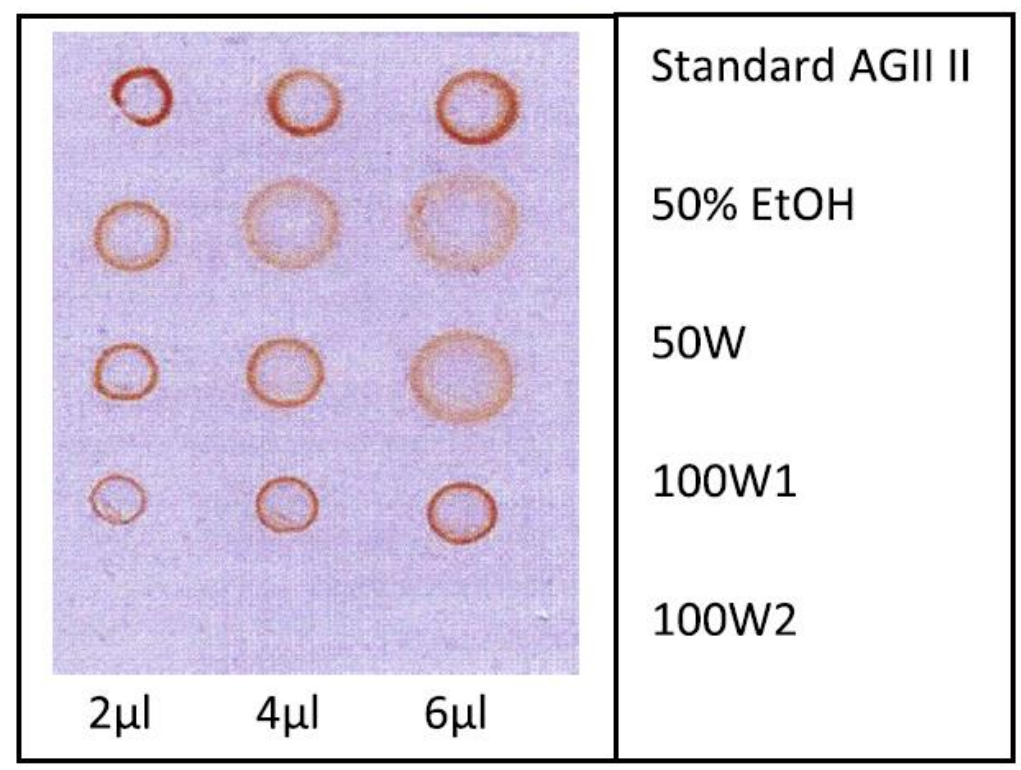

Fig. 2. Yariv reagent precipitation of the fractions. The $100 \mathrm{~W} 2$ did not precipitate. (For interpretation of the references to color in this figure legend, the reader is referred to the web version of this article.)

Furthermore, the polysaccharides contained 1,4-linked xylose units which were attached to galacturonic acid, in addition to 1,4-linked galactose and terminal glucuronic acid units. Artemisia absinthium was recently studied by Correa-Ferreia et al. [7], and the basic structure of the major polysaccharide was determined to be of the arabinogalactan type II. The arabinose units were present as terminal units, 1,2-, 1,5- and 1,3,5- linked units, which is also common in arabinogalactan type II polymers [10]. Methylation analysis of the polymer also provided evidence for the presence of 1,4-linked galacturonic acid residues, and 1,2,4- and 1,3-linked rhamnose units. On the basis of the foregoing discussion, it may be deduced that Artemisia afra contains polysaccharides with similar basic structural features as other Artemisia species reported to date.

\subsection{Biological activities of the polysaccharides from A. afra}

One of the main methods for in vitro determination of the effect of chemical compounds on the immune system is the complement assay [16]. The method used in our laboratories is not influenced by possible contamination with lipopolysaccharides. Thus, the effects observed are an indication of an immunomodulating effect, which may be either stimulating or depressing. Following extraction of the plant material, the effect in the complement assay was the measure by which fractions were selected for further studies. It was found that the biological activities of the selected fractions were higher than those of the standard used (Fig.3), and after purification using gel filtration the activity was still relatively high compared to both the standard and other polysaccharides previously studied in our laboratories thus far.

Fig. 3 shows that fraction $50 \mathrm{~W}$ and 100W1 after gel filtration, were slightly more active than $50 \% \mathrm{EtOH}$, and $100 \mathrm{~W} 2$, while all four were still more active than the standard used. It is 
also interesting to note that the $50 \% \mathrm{EtOH}$ and $50 \mathrm{~W}$ fractions underwent significant loss of activity after gel filtration. This shows that it is not only the highest molecular weight compounds that are active; lower molecular weight (but still higher than $3,5 \mathrm{kD}$ ) water soluble compounds are also of interest for further investigation, but this was not the objective of the present study.

The higher molecular weight fractions obtained after gel filtration chromatography on Sepharose S200 as shown in Fig. 3, being the most active, were also subjected to enzymatic degradation with polygalacturonase. This was to determine which part of the molecule were the most responsible for the bioactivity shown for the purified polymers. After the enzyme treatments, the samples were purified by gel filtration and the different desalted fractions were tested for effects in the complement assay. The results are displayed in Fig. 4.

The activities of fractions from samples 50\% EtOH, 50W and 100W1 are still higher compared to the standard, while 100 W2 shows very low activity (Fig. 4). The monosaccharide composition of the fractions obtained after enzyme degradation is given in Table 1. There is relatively little change in the ratio between the monosaccharide compositions of the parent polymers compared with the most active fraction after enzyme degradation, but they are lower in molecular weight compared to the native polymers. The most striking difference is that 4-O-methy-glucuronic acid is present in all fractions. When comparing the composition of the four fractions, it is evident that fraction $100 \mathrm{~W} 2$ has a very different overall structure than the other three polymers. This is basically a polygalacturonan and has no branches on the galacturonic acid residues. This is based on the results of linkage analyses as given in Table 3 where the major linkage type in this polymer was 1,4 linked galacturonic acid. As we have not removed the possible esters (methyl or acetyl) on the galacturonic acids units, parts of the galacturonic acid chain is not degraded. This sample also has a much lower molecular weight than the other three fractions. Mw may have an influence on the biological activity as obtained in the complement assay. It is also of interest that the other three polysaccharides all contain xylose, and also have branch points on galacturonic acid. It was shown in the polysaccharides from the Malian medicinal tree, Combretum glutinosum that the presence of xylose units attached to the galacturonic acid chain leads to a higher effect in the complement assay, than polysaccharides which are devoid of those substituted galacturonic acid units [10]. The polysaccharides with high bioactivity as found in Lessertia frutescens did also have the feature of xylose units linked to the galacturonic acid chain [36]. In the present study the three most active fractions all have a relatively large arabinogalactan type II part of the structure, while this is very small in fraction $100 \mathrm{~W} 2$. This is evident both from the data presented in Table 2 (linkage analyses), and Fig. 3, the Yariv test, where this fraction gave no precipitation. Several other studies have shown that the AGII structure is important for the effect in the complement assay and the structural requirements have been discussed above. It should also be noted that the polysaccharides present in the leaves of Artemisia princeps also have strong effects in the complement system [33-35]. The studies on the polysaccharides from Artemisia afra clearly show that the water-soluble fraction of this 
plant contains biologically active polysaccharides, and that these have an effect that may be of importance in immune modulation.

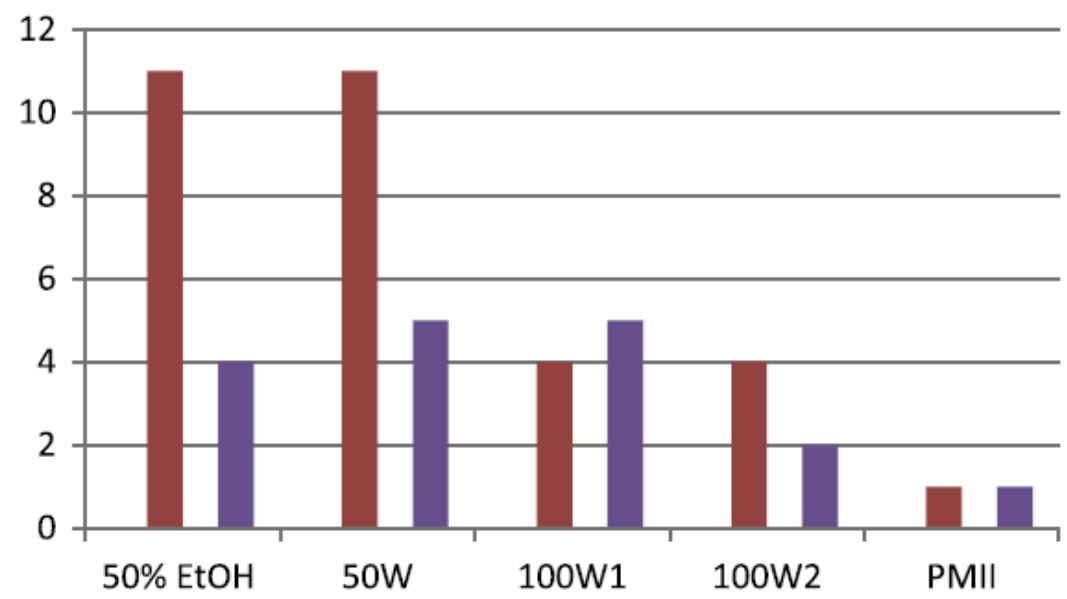

Fig. 3. Effect of the different fractions on the complement fixation test, ICH50 values, related to ICH50 value of the standard polysaccharide, PMII. The values are presented as ICH50 PMII/ICH50 sample. The red bars are the results after anion exchange chromatography and the purple after purification by gel filtration chromatography. (For interpretation of the references to colour in this figure legend, the reader is referred to the web version of this article.)

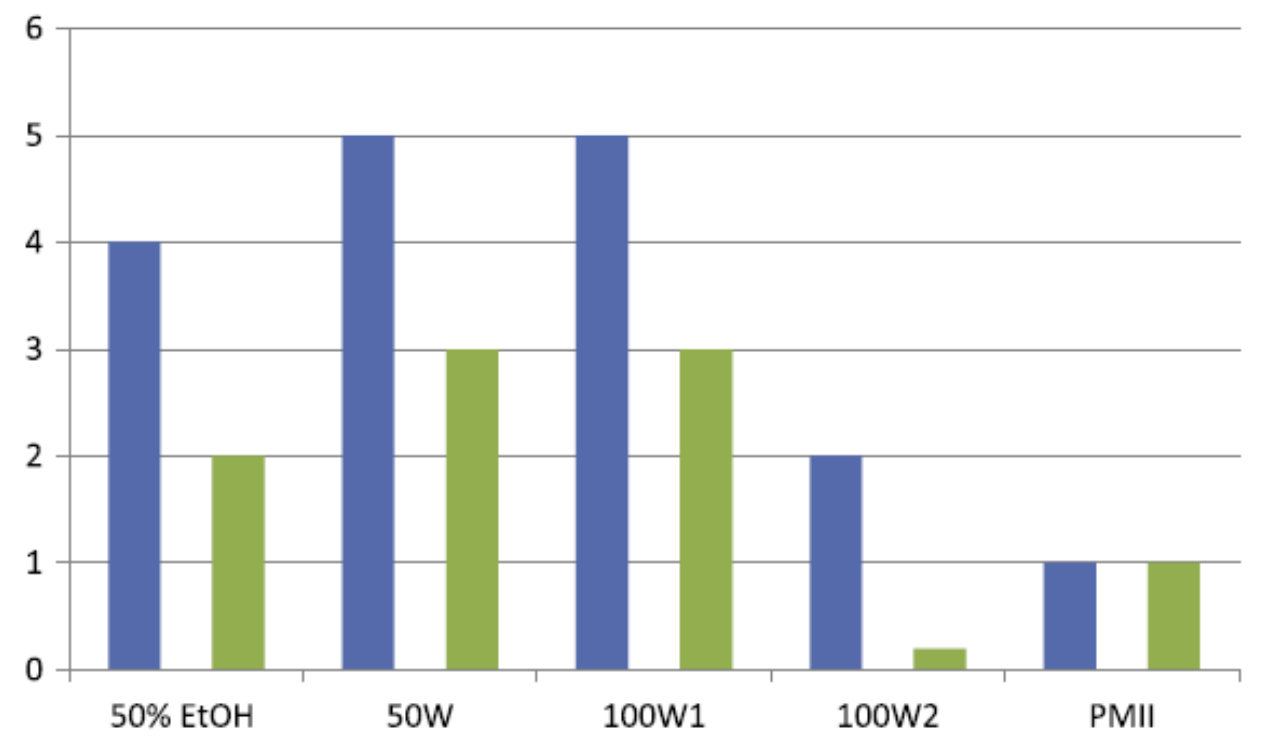

Fig. 4. Effect of the different polysaccharide fractions on the complement fixation test, ICH50 values related to ICH50 value of the standard polysaccharide, PMII. The values are presented as ICH50 PMII/ICH50 sample. The blue bars are the results of the samples after anion exchange and gel filtration chromatography, and the green those with highest activity after separation of polygalacturonase degraded samples following desalting and removal of low molecular weight compounds(in Table 2 denominated 50\% EtOHE, 50WE, $100 \mathrm{~W} 1 \mathrm{E}$ and 100W2E). (For interpretation of the references to colour in this figure legend, the reader is referred to the web version of this article.) 


\section{Conclusion}

Artemisia afra is a plant with several traditional uses as a medicinal plant in South Africa, and some of these uses may influence the immune system. Since the use of plants in traditional medicine often involves water extracts, the polysaccharides present in the water extract of the aboveground plant parts were studied. The studies revealed the presence of pectin like polysaccharides with side chains consisting of arabinogalactan type II structures. Three of the polymers, 50\%EtOH, 50W and 100W1 all contained substitution with xylose on parts of the polygalacturonan chain, which is probably important for bioactivity. The polysaccharides were all tested in the complement assay for immunomodulating properties, and they showed a significant effect in the in vitro complement assay, thus possibly justifying the use of this plant as a medical remedy in traditional African medicine.

\section{Conflict of interest}

The authors declare no conflict of interest.

\section{Acknowledgements}

We are indebted to the South African National Research Foundation

(Grant No. UID65216) for funding for carrying out these studies. 


\section{References}

[1] O.T. Asekun, D.S. Grierson, A.J. Afolayan, Variations in the quality and yield of the essential oil from Artemisia afra using different drying methods, J. Essent. Oil Bear. Plants 10 (1) (2007) 5-9.

[2] Asfaw, N., Licence, P., Novitskii, A.A.; Poliakoff,. M. 2005. Green chemistry in Ethiopia: the cleaner extraction of essential oils from Artemisia afra: a comparison of clean technology with conventional methodology Green Chem., 7(5), 352-356.

[3] A.O.T. Ashafa, T.R. Pitso, Effects of microwave drying on the yield and chemical composition of essential oils of Artemisia afra Jacq. Ex wild from the eastern free state South Africa, J. Essent.Oil Bear. Plants 17 (6) (2014) 1087-1093.

[4] I. Austarheim, B.E. Christensen, I.K. Hegna, B.O. Petersen, J.O. Duus, R. Bye, T.E. Michaelsen, D. Diallo, M. Inngjerdingen, B.S. Paulsen, Chemical and biological characterization of pectin-like polysaccharides from the bark of the Malian med- icinal tree Cola cordifolia, Carbohydr. Polym. 89 (1) (2012) 259-268.

[5] H. Barsett, B.S. Paulsen, Y. Habte, Further characterization of polysaccharides in seeds from Ulmus glabra Huds, Carbohydr. Polym. 18 (2) (1992) 125-130.

[6] R.E. Chambers, J.R. Clamp, An assessment of methanolysis and other actors used in the analysis of carbohydrate containing materials, Biochem. J. 125 (1971) 10091018.

[7] M.L. Correa-Ferreira, G.R. Noleto, C.L.O. Pekowicz, Artemisia absinthium and Artemisia vulgaris: a comparative study of infusion polysaccharides, Carbohydr. Polym. 102 (2014) 738-745.

[8] M. Dubois, K.A. Gilles, J.K. Hamilton, P.A. Rebers, F. Smith, Colorimetric method for determination of sugars and related substrances, Anal. Chem. 28 (1956) 350-356.

[9] J.R. Dunkelberger, W.C. Song, Complement and its role in innate and adaptive immune responses, Cell Res. 20 (2010) 34-50.

[10] S. Glaeserud, T.E. Groenhaug, T.E. Michaelsen, M. Inngjerdingen, H. Barsett,

D. Diallo, B.S. Paulsen, Immunomodulating polysaccharides from leaves of the Malian medicinal tree Combretum glutinosum; structural differences between small and large leaves can substantiate the preference for small leaves by some healers, J. Med. Plants Res. 5 (13) (2011) 2781-2790.

[11] A.N. Guantai, I. Addae-Mensah, Cardiovascular effect of Artemisia afra and its constituents, Pharm. Biol. 37 (5) (1999) 351-356.

[12] I.A. Issa, H.M. Bule, Hypoglycemic Effect of Aqueous and Methanolic Extract of Artemisia afra on Alloxan Induced Diabetic Swiss Albino Mice. Evidence-based complementary and alternative medicine, (2015) (2015752486).

[13] J.B. Kim, N.C. Carpita, Changes in esterification of uronic acid groups of cell wall polysaccharides during elongation of maize coleoptiles, Plant Physiol. 98 (1992) 646653.

[14] K. Kitazawa, T. Tryfona, Y. Yoshimi, Y. Hayashi, S. Kawauchi, L. ANtonov, H. Tanaka, T. Takahashi, S. Kaneko, P. Dupree, Y. Tsumuraya, $\beta$-GAlactosyl Yariv reagent binds to the $\beta-1,3$ galactan of arabinogalactan proteins, Plant Physiol. 161 (2013) 1117-1126. 
[15] N.Q. Liu, F. Van der Kooy, R. Verpoorte, Artemisia afra: a potential flagship for African medicinal plants? S. Afr. J. Bot. 75 (2009) 185-195.

[16] T.E. Michaelsen, A. Gilje, A.B. Samuelsen, K. Høgåsen, B.S. Paulsen, Interaction between human complement and a pectin type polysaccharide fraction, PMII, from the leaves of Plantago major L, Scand. J. Immunol. 52 (2000) 483-49o.

[17] S. Ntutela, P. Smith, L. Matika, J. Mukinda, H. Arendse, N. Allie, D.M. Estes, W. Mabusela, P. Folb, L. Steyn, Q. Johnson, W.R. Folk, J. Syce, M. Jacobs, Efficacy of Artemisia afra phytotherapy in experimental tuberculosis, Tuberculosis 89 (S 1) (2009) S33-40.

[18] A.O. Oyedeji, A.J. Afolayan, A. Hutchings, Compositional variation of the essential oils of Artemisia afra Jacq. from three provinces in South Africa - a case study of its safety, Nat. Prod. Commun. 4 (6) (2009) 849-852.

[19] B.S. Paulsen, H. Barsett, Bioactive pectic plysaccharides, Adv. Polym. Sci. 186 (2005) 69-101.

[20] B.S. Paulsen, D.J. Craik, D.E. Dunstan, B.A. Stone, A. Bacic, The Yariv reagent: behaviour in different solvents and interaction with a gum arabic arabinogalactanprotein, Carbohydr. Polym. 106 (2014) 460-468.

[21] L. Spies, T.C. Koekemoer, A.A. Sowemimo, E.D. Goosen, M. van de Venter, Caspase- dependent apoptosis is induced by Artemisia afra Jacq. ex Willd in a mitochondria- dependent manner after G2/M arrest, S. Afr. J. Bot. 84 (2013) 104109.

[22] T.O. Sunmonu, A.J. Afolayan, Evaluation of antidiabetic activity and associated toxicity of Artemisia afra aqueous extract in Wistar rats, Evid. Based Complement. Alternat. Med. 2013929074 (2013).

[23] D.P. Sweet, R.H. Shapiro, P. Albersheim, Quantitative analysis by various GLC [gas- liquid chromatography] response-factor theories for partially methylated and partially ethylated alditol acetates, Carbohydr. Res. 40 (2) (1975) 217-225.

[24] Togola, A., Inngjerdingen, M., Diallo, D., Barsett, H., Rolstad, B., Michaelsen, T.E., Paulsen, B,S. 2008. Polysaccharides with complement fixing and macrophage sti- mulatiion activit from Opilia celtidifolia, isolation and partia characterisation. J. Ethnopharmacol., 115, 423-431.

[25] G.W.B. Van der Lingen, Oil of Artemisia afra. Perfumery and essential oil, Theatr.

Rec. 21 (1930) 473.

[26] G.-J. Van Holst, E.E. Clarke, Quantification of Arabino-galactan-protein in plant extracts by single radial gel-diffusion, Anal. Biochem. 148 (1985) 446-450.

[27] L. Venables, T.C. Koekemoer, M. Van de Venter, E.D. Goosen, Isoalantolactone, a sesquiterpene lactone from Artemisia afra Jacq. ex Willd and its in vitro mechanism of induced cell death in HeLa cells, S. Afr. J. Bot. 103 (2016) 216-221. [28] A.M. Viljoen, S.F. van Vuuren, T. Gwebu, B. Demirci, K.H.C. Baser, The geographical variation and antimicrobial activity of African wormwood (Artemisia afra Jacq.) essential oil, J. Essent. Oil Res. 18 (2006) 19-25 Spec. Ed..

[29] Von Koenen, E. 2001, Medicinal, poisonous and edible plants in Namibia, Klaus Hess Publishers, 2001, Windhoek - Göttingen. 
[30] H. Wangensteen, D. Diallo, B.S. Paulsen, Medicinal plants from Mali: chemistry and biology, J. Ethnopharmacol. 176 (2015) 429-437.

[31] Van Wyk, B.-E., van Oudtshoorn, B., Gericke, N. 2002. Medicinal Plants of South Africa, 2nd ed. Briza Pblications, Pretoria, South Africa.

[32] H. Yamada, H. Kiyohara, "Comprehensive Glycoscience: From Chemistry to Systems Biology", Immunomodulating Activity of Plant Polysaccharide Structures, Elsevier Ltd, Amsterdam, 2007.

[33] H. Yamada, K. Ohtani, H. Kiyohara, J.C. Cyong, Y. Otsuka, O. Yoshio, Purification and chemical properties of anti-complementary polysaccharides from the leaves of Artemisia princeps, Planta Med. 51 (2) (1985) 121-125.

[34] H. Yamada, Y. Otsuka, S. Omura, Structural studies on anti-complementary polysaccharides from the leaves of Artemisia prínceps, Planta Med. 52 (4) (1986) 311-314.

[35] H. Yamada, H. Kiyohara, Y. Otsuka, Further structural studies of an anti-complementary acidic heteroglycan from the leaves of Artemisia prínceps, Carbohydr. Res. 170 (1987) 181-191.

[36] B.-Z. Zhang, W.K. Leung, Y.F. Zou, W. Mabusela, Q. Johnson, T.E. Michaelsen, B.S. Paulsen, Immunomodulating polysaccharides from Lessertia frutescens leaves: isolation, characteri $\mathrm{n}$ and structure activity relationship, J. Ethnopharmacol. 152 (2) (2014) 340 